\title{
Le succès d'édition des œuvres de Benito Pérez Galdós: essai de bibliométrie (I)
}

\author{
JEAN-FRANÇOIS BOTREL \\ Universidad de Rennes II, Haute-Bretagne
}

De la compra y venta de libros no digas una palabra, que esa monserga mercantil a nadie le interesa". A lo que respondí: "Contra lo que me ha dicho mi ninfa gentil, opino yo que el mecanismo in terno de la producción literaria despierta en el público interés más vivo que la producción misma.

Benito Pérez Galdós Memorias de un desmemoriado, Madrid, C. I. A. P., 1930, pp. 218-219 En Galdós lo que predomina es la obra total, el conjunto, la masa.

Miguel de Unamuno

(El Liberal, Madrid, 21-II-1920)

\section{LA MESURE DU SUCCÈS ${ }^{1}$}

1.1. Succès d'édition et succès. Les succès d'édition d'une oeuvre, c'est-à-dire la quantité d'exemplaires du livre mis en circulation et vendus est un indicateur du succès tout court. Celui-ci

1 Liste des principales œuvres de Benito Pérez Galdós (Bibliografía de Galdós, I, Gran Canarias, Cabildo, 1972).

\section{Episodios Nacionales}

Primera serie: 1. Trafalgar (1873), 2. La Corte de Carlos IV (1873), 3. El 19 de marzo y el 2 de mayo (1873), 4. Bailén (1873), 5. Napoleón en Chamartín (1873), 6. Zaragoza (1874), 7. 


\section{n'est pas seulement, comme disait Gaston Rageot ${ }^{2}$, «le fait qu'une œuvre produite par une personnalité a été adoptée par une collectivité», mais aussi le degré de réception et d'estime par le public.}

\section{Entre le succès d'édition d'un livre et le succès, tout court, d'une oeuvre, il est évidęnt qu'il ne peut exister d'équivalence}

Gerona (1874), 8. Cádiz (1874), 9. Juan Martín el Empecinado (1874), 10. La batalla de los Arapiles (1875).

Segunda serie: 11. El equipaje del rey José (1875), 12. Memorias de un cortesano... (1875), 13. La segunda casaca (1876), 14. El Grande Oriente (1876), 15. Siete de Julio (1876), 16. Los cien mil hijos de San Luis (1877), 17. El terror de I824 (1877), 18. Un voluntario realista (1878), 19. Los apostólicos (1879), 20. Un faccioso más y algunos frailes menos (1879).

Tercera serie: 21. Zumalacárregui (1898), 22. Mendizábal (1898), 23. De Oñate a la Granja (1898), 24. Luchana (1899), 25. La campaña del Maestrazgo (1899), 26. La estafeta romántica (1899), 27. Vergara (1899), 28. Montes de Oca (1900), 29. Los Ayacuchos (1900), 30. Bodas reales $(1900)$.

Cuarta serie: 31. Las tormentas del 48 (1902), 32. Narváez (1902), 33. Los duendes de la camarilla (1903), 34. La revolución de julio (1904), 35. O'Donnell (1904), 36. Aita-Tettauen (1905), 37. Carlos VI en la Rápita (1905), 38. La vuelta al mundo en la «Numancia» (1906), 39. Prim (1906), 40. La de los tristes destinos (1907).

Quinta serie: 41. España sin rey (1908), 42. España trágica (1909), 43. Amadeo I (1910), 44. La primera República (1911), 45. De Cartago a Sagunto (1911), 46. Cánovas (1912).

Novelas

Novelas de la primera época: 1. La Fontana de Oro (1870), 2. La sombra (1871), 3. El audaz (1871), 4. Doña Perfecta (1876), 5. Gloria (1877), 6. Marianela (1878), 7. La familia de León Roch (1878).

Novelas españolas contemporáneas: 8. La desheredada (1881), 9. El amigo Manso (1882), 10. El doctor Centeno (1883), 11. Tormento (1884), 12. La de Bringas (1884), 13. Lo prohibido (1884-1885), 14. Fortunata y Jacinta (1886-1887), 15. Miau (1888), 16. La incógnita (1889), 17. Torquemada en la hoguera (1889), 18. Realidad (1889), 19. Angel Guerra (1891), 20. Tristana (1892), 21. Torquemada en la Cruz (1893), 21. La loca de la casa (1893), 22. Torquemada en el Purgatorio (1894), 23. Torquemada y San Pedro (1895), 24. Nazarin (1895), 25. Halma (1895), 26. Misericordia (1897), 27. El abuelo (1897), 28. Casandra (1905), 29. El caballero encantado (1909), 30. La razón de la sinrazón (1915).

\section{Teatro}

2. Realidad (1892), 3. La loca de la casa (1893), 4. Gerona (1893), 5. La de San Quintín (1894), 6. Los condenados (1895), 7. Voluntad (1896), 8. Doña Perfecta (1896), 9. La fiera (1897), 10. Electra (1901), 11. Alma y vida (1902), 12. Mariucha (1903), 13. El abuelo (1904). 14. Bárbara (1905), 15. Amor y ciencia (1905), 16. Pedro Minio (1908), 17. Zaragoza (1908), 18. Casandra (1910).

2 Le succès. Auteurs et public. Essai de critique sociologique, Paris. Félix Alcan éditeur. 1906. p. 9. 
arithmétique: un livre acheté n'est pas toujours lu; pour lire une œuvre, il n'est pas toujours besoin d'acheter le livre; le succès d'une oeuvre dramatique se mesurera davantage par le taux de fréquentation que par le nombre d'exemplaires vendus; le succès d'un poème déterminé et celui du livre qui le contient peuvent très bien ne pas coïncider, etc.

Mais pour apprécier le succès d'un roman de façon non subjective, la donnée fondamentale est le calcul du comportement commercial du livre et son observation à l'aide d'une statistique appropriée: la bibliométrie.

1.2. La bibliométrie, à certaines conditions. Le succès d'édition peut être mesuré à court, moyen et long terme, avec une exactitude et une fiabilité plus ou moins grandes, à condition de disposer d'informations de première main, telles que les archives des maisons d'édition, les livres de comptabilité de libraires, etc., les plus complètes possible et sûres.

Pour l'étude du succès d'édition des oeuvres de Benito Pérez Galdós (Galdós dorénavant), on dispose d'une série d'informations abondantes et aussi fiables que possible, tirées des archives du troisième éditeur de Galdós, la maison Hernando, ou publiées par M. Guimerá Peraza dans son étude sur Maura y Galdós ${ }^{3}$, en plus des indications de tirage sur les couvertures et pages de titre à partir de 1898 et 1904, selon les oeuvres, et de la fondamentale bibliographie de Galdós par Manuel Hernández Suárez, déjà citée.

A partir de ces données brutes ou élaborées, présentées dans les documents $\mathrm{n} .^{\circ} 1$ à $10^{4}$, on peut essayer de mesurer le succès d'édition de la plupart des oeuvres qui composent l'opus galdosien (Episodios nacionales, Novelas de la primera época, Novelas españolas contemporáneas) et, accessoirement dans notre cas, oeuvres pour le théâtre.

3 Ediciones del Excmo. Cabildn Insular de Gran Canaria. 1967.

4 Ver la segunda parte de este estudio en el próximo número de Anales de Literatura Española. 
Les résultats absolus des ventes d'exemplaires par livre ou par série depuis leur première édition jusqu'en juillet 1973, et par sous-périodes, permettent de calculer des taux indices basés sur les rythme mensuel. On peut ainsi estimer les différents niveaux de consommation de livres, l'évolution générale ou le comportement de l'opus galdosien sur une période qui varie entre 100 et 30 ans selon les oeuvres, les variations conjoncturelles et aussi les disparités internes entre les différents genres, les différentes séries d'Episodios nacionales, ou même les différentes oeuvres.

1.3. La réception de l'oeuvre de Galdós. Cette observation du comportement de l'oeuvre, influencé par des facteurs para ou extra-littéraires dont il conviendra d'estimer le poids, ne doit pas s'arrêter à un néo-positivisme périmé; elle doit s'articuler sur une recherche des éléments constitutifs du succès: les uns sont extérieurs à l'oeuvre et plus conjoncturels (fonction socioidéologique de l'oeuvre) et d'autres sont plus structuraux (fonction socio-esthétique) et c'est leur jeu dialectique dans le processus de la communication qui explique les disparités et les fluctuations de la cote des oeuvres de Galdós depuis la Première République jusqu'aux dernières années du franquisme et peut-être aussi l'inégale et variable actualité d'un message qui, dans quelques cas, est si atemporel et universel qu'il en devient classique.

\section{BASES ET LIMITES D'UNE BIBLIOMÉTRIE}

2.1. Des sources d'archives à compléter. Comme on le sait, à partir du 15 janvier 1904, la maison d'édition Obras de Pérez Galdós créée par Galdós à la suite de sa séparation d'avec Miguel Honorio de Cámara cesse officiellement de fonctionner et confie l'administration de son fonds, c'est-à-dire les oeuvres de Galdós, à la maison d'édition Perlado, Páez y Cía., Sucesores de Hernando qui a continué à les administrer, puis à les éditer, sous la raison sociale Hernando, S.A., jusqu'à aujourd'hui. 
Dans les archives de cette maison sont conservés des documents ${ }^{5}$ qui permettent d'observer l'évolution du comportement éditorial des oeuvres de Galdós de 1904 à 1906 et de 1941 à 1973, ainsi que, dans une certaine mesure, de 1906 à 1908-1909 et durant la Seconde République. Les données brutes puisées à cette source sont recueillies dans les documents $n .{ }^{\circ} 1$ à 6 .

Pour la période antérieure à 1904, l'absence de données suivies pour les années 1873 à 1897 provient du caractère apparemment lacunaire des archives de la société d'édition La Guirnalda, conservées à la Casa Museo Pérez Galdós de Las Palmas; cela représente un obstacle, pour le moment insurmontable, pour l'étude de la première réception des deux premières séries d'Episodios nacionales et de 25 des 30 ou 31 autres romans.

Sur la période comprise entre le 22 mai 1897 et le 15 janvier 1904 durant laquelle Galdós fut son propre éditeur, on dispose des informations publiées par Marcos Guimerá Peraza relatives à l'état des stocks en mai 1897 et accessoirement en novembre $1896^{6}$.

Des trois périodes, seules les deux dernières peuvent donc faire l'objet d'une véritable bibliométrie, parce que le caractère parfois lacunaire des données peut être compensé ou corrigé par le comptage des éditions successives et du nombre d'exemplaires de chacune de celles-ci d'après les chiffres de tirage, imprimés sur les couvertures ou les pages de titre et recueillis par Manuel Hernández Suárez dans sa Bibliografía de Galdós.

2.2. Les chiffres de tirage: leur fiabilité et l'inflation. L'indication du nombre de milliers d'exemplaires imprimés, puis des milliers cumulés a été une initiative de la maison Obras de Pé-

5 En voici la liste: -Nota detallada de las obras recibidas de D. B. P. Galdós (15-01-1904); -Inventario del 7-05-1906; -Extracto de la cuenta de B. P. Galdós contra los Sres. Perlado, Páez y Cía. desde 12-06-09 a 21-01-1910; - Liquidación del adelanto de 52.526 ptas. 80 cents. hecho a D. B.P. Galdós en 22-06-07 con arreglo a contrato (Madrid 12-06-1909), Registro de trabajos entregados a la Imprenta, Libro II, 1-09-1930 a 4-04-1939, et d'autres documents contemporains.

Je remercie José-Luis Perlado Páez de Valluerca, directeur-gérant de la Casa editorial Hernando, S.A., pour son esprit de collaboration et son chaleureux accueil en juillet 1973.

6 Op. rit., pp. 153-156. 
rez Galdós -c'est même sans doute une innovation pour l'Espagne- à l'occasion de la publication de la Troisième série des Episodios nacionales. Cette mention figure ensuite sur la Quatrième série des Episodios, puis sur l'édition de 1899 de Doña Perfecta et sur celles de Gloria, Marianela, La Familia de León Roch, etc.

En 1904, Perlado, Páez y Cía. suivent cette pratique en l'étendant même, mais a posteriori, à l'ensemble de l'oeuvre alors publiée, c'est-à-dire en précisant (en fait en fixant), à l'occasion des rééditions des deux premières séries d'Episodios, des romans et des oeuvres pour le théâtre faites par leurs soins, le nombre total d'exemplaires imprimés depuis la première édition de chaque oeuvre. Ainsi, par exemple, la 5e édition de Tormento, en 1906, porte les chiffres 15.000 puis 16.000 , ce qui veut dire, sans nul doute, que de 1884 à 1906 on a publié un total de 16.000 exemplaires.

Se pose bien sûr le problème de la fiabilité de ces chiffres, établis on ne sait selon quelles informations (celles fournies par Galdós lui même?) ni selon quels critères. Sont-ils exacts, approximatifs, arbitraires voire fantaisistes?

En divisant le nombre des exemplaires indiqué sur les éditions immédiatement postèrieures à 1904, par le nombre total des éditions, on obtient cependant pour chaque édition un chiffre vraisemblable: une moyenne de 5.000 pour chaque titre des deux premières séries d'Episodios et de 4.000 pour les romans ${ }^{7}$, chiffre

7 Il s'agit bien sûr d'une moyenne et l'on sait, par exemple, grâce à Jacques Beyrie (Galdós et son mythe, Paris, Lille, 1980, pp. 168-169), que les premières éditions de Trafalgar et de $L a$ Corte de Carlos $I V$ n'ont été que de 1.000 et 3.000 exemplaires respectivement, et que ce n'est qu'après qu'on eût constaté le plaisir avec lequel le public avait reçu $L a$ Corte de Carlos I ${ }^{\prime}$ qu'on fit un tirage de 4.000 exemplaires pour El 19 de marzo..., qui allait être désormais, le tirage ordinaire.

En 1906-1909, les rééditions des Episodios ou des romans sont presque toutes de 4.000 exemplaires et les premières éditions des auvres nouvelles doivent être, selon la clause $6 \mathrm{du}$ contrat du 7-05-1906, de 16.000 exemplaires, s'il s'agit d'Episodios, et de 8.000 maximum, s'il s'agit de romans ( $c f$. J.-F. Botrel, «Galdós y la Casa editorial Perlado, Páez y Cía. Sucesores de Hernando (1904-1920)", Letras de Deusto, vol. 4, núm. 8, julio-diciembre 1974. pp. 265266.

Le 31-12-1920, les héritiers de Galdós, María Pérez Galdós et Juan Verde, exigent, dans la clause 2 du contrat proposé à Hernando, des éditions de 4.000 exemplaires au moins pour les Episodios, de 2.000 pour les romans et de 1.000 pour le théâtre. 
assez conforme aux pratiques éditoriales de l'époque ${ }^{8}$, aux témoignages sur le succès de Galdós et de ses oeuvres alors, et au fait que les rééditions étaient faites selon le procédé très souple et très rapide de la stéréotypie ${ }^{9}$.

En attendant que l'accès aux archives de M. H. de Cámara permette (peut-être) d'établir des chiffres définitifs, nous prenons ces chiffres pour des bases vraisemblables pour la période 1873-1897.

Pour les années 1907-1908, les indications de tirage selon les archives Hernando permettent de vérifier l'exactitude des chiffres indiqués sur la couverture, sans qu'on sache, en revanche, quand ces tirages ont été mis sur le marché, car il semble qu'on avait l'habitude d'imprimer la totalité de l'édition (4.000 exemplaires pour les Episodios), mais de fragmenter leur distribution avec une couverture différente pour chaque sous-édition de 2.000 exemplaires, comme dans le cas de l'édition de 4.038 exemplaires de Zumalacárregui faite le 5-07-1909 qui se traduit sur les couvertures par les chiffres 16.000 et 18.000 . Pour les romans, édités à 2.000 exemplaires, cela se traduit par des chiffres qui vont de 1.000 en 1.000. Le même procédé est encore employé entre 1931 et 1939 .

Jusqu'en 1920, l'utilisation des chiffres mentionnés sur les couvertures ne pose pas trop de problèmes, même si l'on peut supposer qu'il a existé pour certaines oeuvres des éditions intermédiaires, en particulier vers 1916-1917, non recensées par Manuel Hernández Suárez.

Après 1920 , il se produit une brusque et forte augmentation dans les chiffres indiqués, surtout sur la Première série d'Episodios (de 51.000 en 1914 à 66.000 en 1927 pour Trafalgar, par

8 La première édition de $S u$ único hijo de Leopoldo Alas est, par exemple, prérue. en 1889. à 6.000 exemplaires ( $c f$. Clarín y sus editores, Rennes, Université de Haut Bretagne. 1981. p. 47), et les Obras completas de Pereda sont tirées à 5.000 exemplaires.

9 Sur ce point, voir M. Guimerá Peraza, op. cit., p. 125 et le contrat du 7-05-1906, clause 7 (apud J. - F. Botrel, «Galdós y la Casa editorial...», loc. (it., p. 266). 
exemple). Ce qui cache sans doute une édition intermédiaire vers 1920 non répertoriée, mais étant donné les circonstances favorables, qui seront évoquées plus loin, le rythme de vente des œuvres de Galdós ne semble pas, dans le cas présent, influencé par une quelconque imprécision ou une inflation volontaires de la part de l'éditeur ${ }^{10}$.

Entre 1931 et 1939, le livre d'imprimerie de la maison Hernando (Registro de imprenta) fournit à nouveau des chiffres précis qui coïncident, dans la quasi-totalité des cas, avec ceux imprimés sur les couvertures ${ }^{11}$.

Après 1940, l'examen comparatif des chiffres sur les tirages conservés dans les Archives Hernando et ceux indiqués sur les couvertures permet de rétablir une vérité très falsifiée - les éditeurs eux-mêmes le reconnaissent - qui a donné lieu à des estimations exagérées sinon fantaisistes sur la diffusion des oeuvres de Galdós. Un ou deux exemples nous serviront de garde-fou pour l'avenir: Doña Perfecta, dont la dernière édition connue, celle de 1922 , portait le chiffre 42.000 , se retrouve à 100.000 ou 100.500 en mai 1942, date á laquelle est faite une édition de 4.959 exemplaires! Quant aux Episodios nacionales, le chiffre des exemplaires publiés est uniformisé à 100.000 pour tous les titres de la Première série, alors que, par exemple, Mendizábal n'atteignait, en juin 1934, qu'un tirage cumulé de 34.000 exemplaires! Ces 66.000 exemplaires octroyés sont bien sûr totalement imaginaires. Il convient donc de rétablir les véritables chiffres cumulés. C'est ce qui est fait dans les documents $n .^{\circ} 9$ et 10 où, pour chaque édition recensée est indiqué le chiffre maximun connu des exemplaires cumulés.

2.3. L'aide de la science bibliographique. Enfin, comme élément complémentaire, l'évolution chronologique des édi-

10 Les livres d'imprimerie correspondant à cette époque ont disparu durant la guerre civile. à ce qu'il m'a été dit.

11 La comparaison entre les chiffres du Registro de Imprenta et ceux des couvertures permet de constater que la maison Hernando tire en une seule fois de 4.000 à 6.000 exemplaires d'un volume d'E. N., par exemple, mais qu'elle les commercialise millier après millier avec des couvertures portant mention de cette progression. 
tions ${ }^{12}$ des Episodios et des romans (documents 7 et 8) permet de couvrir la période la moins documentée et d'observer des politiques d'édition très différentes selon les oeuvres et révélatrices d'une diffusion/réception inégale et variable ${ }^{13}$.

Avec de telles bases, on peut essayer de procéder à une bibliométrie qui n'est certes pas totalement exhaustive ni rigoureuse, mais qui n'est pas non plus totalement arbitraire.

2.4. La détermination de l'unité de mesure. En effet, on peut alors calculer le nombre total d'exemplaires publiés ${ }^{14}$ et mesurer, par rapport à d'autres opera, l'impact global de l'opus galdosien; mais surtout, en utilisant une unité de mesure standardisée, apprécier le succès relatif des différents genres, des différentes séries ou oeuvres dans la longue durée (de 1873 à 1973, dans le cas extrême de Trafalgar) ou durant des sous-périodes: 18701897, 1897-1904, Seconde République, Franquisme, etc.) et même, parfois, d'une année sur l'autre.

12 Le nombre d'exemplaires, même à l'époque, est variable, c'est pourquoi le fait que El Escándalo de P. A. de Alarcón ait connu 38 éditions jusqu'en 1943 et El Sombrero de tres picos 30, ne veut pas dire a priori qu'on ait publié ni vendu plus d'exemplaires de ces œuvres que de Trafalgar ou Marianela qui, en 1973, n'en sont encore qu'à leur 22ème et 21 ème édition, respectivement.

13 On voit ainsi facilement que, de 1879 à 1896 , on a fait beaucoup plus éditions d'Episodios (50 pour 25 titres) ou de Novelas de la primera época (25 éditions) que de Novelas españolas contemporáneas ( 26 pour 18 titres). Le monde d'impression (stéréotypie à coup sûr pour les Episodios) n'est certainement pas étranger à cette politique.

14 Pour les calculs ou les estimations, il n'est tenu compte que des éditions faites par M. H. de Cámara, Galdós lui-même et Perlado, Páez y Cía qui devient ensuite la Casa editorial Hernando $S$. A. Sont exclues par conséquent les éditions faites après 1940 par Aguilar (Obras completas et Colección Crisol), les éditions sud-américaines (Losada, Espasa-Calpe Argentina, etc., certains d'entre elles plus clandestines qu'autorisées); l'édition illustrée des deux premières séries des Episodios nacionales (voir la note au document $n .^{\circ}$ ) qui fut d'ailleurs un grave échec comercial; les premières publications dans des revues ou des journaux (comme celle de Doña Perfecta dans la Revista de España), ainsi que des adaptations comme les Episodios à l'usage des enfants (Episodios nacionales, Guerra de la Independencia extractada para uso de los ninos - 13.000 exemplaires publiés de 1906 à 1916-17 - cf. Alberto Navarro González, Los Episodios Nacionales extractados por Galdós, "Actas del primer congreso internacional gadosiano", Las Palmas, 1977, p. 167) ou Los Episodios nacionales por Don Benito Pérez Galdós narrados a los niños por su hija Doña María Pérez Galdós.

Toutes ces éditions non prises en compte, bien que certaines aient été importantes (la première édition des Obras completas de Aguilar a eu, selon le Registro general de la Propiedad intelectual, un tirage de 12.500 exemplaires et elle en sont, en 1965, à la neuvième édition), nous amènent à considérer les rythmes obtenus comme des rythmes minimum. 
L'unité de mesure retenue est le nombre moyen d'exemplaires vendus par mois, soit le rythme de vente, facile à obtenir en divisant le nombre total d'exemplaires vendus durant la période considérée ${ }^{15}$ par le nombre de mois compris dans la dite période, en partant du principe, vérifié, qu'on ne fait guère un nouveau tirage d'un livre avant que le précédent ne soit épuisé ou en passe de l'être ${ }^{16}$.

Cette unité de mesure est totalement précise, au niveau des calculs, lorsqu'il s'agit de périodes bien délimitées (du 15 janvier 1904 au 7 mai 1906, par exemple, c'est-à-dire 28 mois) et plus approximative quand les seules bases de référence sont les années (1877 à 1932, par exemple).

Cette marge d'imprécision à laquelle s'ajoutent d'autres risques d'erreur ${ }^{17}$ a pour conséquence que les différences dans les rythmes de vente, pour être significatives, doivent présenter un écart assez important, estimé à cinq points. Ainsi le fait que le rythme mensuel de vente de Torquemada en la cruz soit de 19 (exemplaires) de 1893 à 1936 et celui de Tristana de 17 de 1892 à 1932 ne permet pas d'établir que le premier roman a connu un plus grand succès, même à long terme, que le second.

L'ensemble des variations et écarts dans les rythmes est représenté dans les documents 11 à 20 , commenté dans la deuxième partie de cette étude et interprété dans la troisième.

15 Pour le calcul du rythme annuel, on prend en compte les années complètes, ce qui peut, en théorie, fausser les résultats de onze mois. Le poids de cette marge d'erreur ets d'autant plus for que la période considérée est plus courte.

16 Il peut cependant y avoir anticipation (c'est le cas, parfois, lorsque Galdós doit régler une dette à son administrateur, Hernando; $c f$. in J. - F. Botrel, «Galdós y la Casa editorial...», loc. cit., la Liquidación... du 12-06-1909 ou l'Extracto de cuenta ... du 21-01-1910), mais cela n'a aucune incidence sur le rythme de vente des exemplaires. Par contre, la destruction pour cause de guerre ou de "quemas ideológicas... en bibliotecas públicas y centros de enseñanzą» après 1939, comme le rappelle Alfonso Armas Ayala dans son «Introducción a las Actas del primer congreso internacional galdosiano...» (p. 10), fausse ce rythme en l'accélérant éventuellement par la suite, par compensation, de même que la non réédition d'un titre peut très bien être due à une décision de type idéologique et non commercial.

17 Ceux dérivés de la multiplication des calculs nécessaires à cette bibliométrie, en particulier. 


\section{COMMENTAIRES}

3.1. Sur la marque de fabrique Benito Pérez Galdós. Le premier commentaire sur les résultats obtenus concerne le caractère massif et durable de la vente des produits «Benito Pérez Galdós».

3.1.1. Le caractère massif des ventes. On appréciera la première caractéristique à l'aide des chiffres suivants: cinquante ans après la parution de La Fontana de Oro, soit l'année de la mort de Galdós, il a été imprimé et mis en circulation quelque 1.700 .000 volumes (1.250.000 d'Episodios nacionales et 450.000 de romans), ce qui donne, compte non tenu des oeuvres pour le théâtre, une moyenne théorique de presque 35.000 volumes par an ${ }^{18}$.

Un chiffre énorme pour l'Espagne à l'époque et que seul, peut-être, a pu dépasser quelqu'un comme Vicente Blasco Ibáñez.

Il faut cependant relativiser et peut-être même rectifier cette première impression en comparant ces chiffres obtenus, avec $\mathbf{7 6}$ livres, par le "puerco menor», comme le qualifiait un quotidien de México ${ }^{19}$, avec ceux du «puerco mayor», Emile Zola, qui pendant la même période (1871-1923), mais avec seulement les 20 romans des Rougon-Macquart, met 2.549 .000 volumes de ses œuvres en circulation ${ }^{20}$.

On peut aussi faire la comparaison avec certains écrivains espagnols. Ainsi Juan Valera affirme qu'en 1904 sa Pepita Jiménez en est à la 19ème édition, avec plus de 40.000 exemplaires publiés

18 Ces estimations sont faites d'après les documents 9 et 10.

19 El Pais, 15-07-1902.

20 Selon A. Lanoux (Préface à Les Rougon Macquart, Paris, Gallimard, 1960, vol. I, pp. XXX$\mathrm{XXXI}$ ). On peut constater que le roman de la série, les moins vendu en 1923 (La conquête de Plassans) se trouve déjà aussi vendu que Gloria en 1935. Il est évident que le public potentiel est en France beaucoup plus nombreux qu'en Espagne, où, á l'image de La Ilustración española y americana le 30 mai 1891 (p. 339), on s'étonne, par exemple, de voir absorbés les énormes tirages de l'œuvre de Victor Hugo. 
depuis $1874^{21}$. Or Doña Perfecta, publié en 1875 , n'atteint, en 1905 que 33.000 exemplaires...

De même les romans de José María de Pereda ont finalement eu des rythmes de vente supérieurs, en moyenne, à ceux de Galdós: Peñas arriba est au même niveau que le plus vendu des romans de Galdós (Marianela) et onze autres de ses romans se situent largement au dessus des Novelas españolas contemporáneas de Galdós ${ }^{22}$.

Ce qui est exceptionnel chez Galdós, ce n'est donc pas la vente record de tel ou tel titre (en six mois, Pequeñeces, du Padre Coloma, est deux fois plus vendu que Fortunata y Jacinta en 20 ans!), c'est le niveau moyen de ses ventes, supérieur à celui de la plupart des écrivains de l'époque, qui, multiplié par le grand nombre de ses oeuvres, donne cette impression de masse, si bien perçue par Unamuno.

On comprend mieux ce dernier lorsqu'on se rappelle qu'en 1899 les 1.500 exemplaires de Paz en la guerra sont à peine épuisés, alors que, à cette même date, on a déjà tiré au moins 6.000 exemplaires de Misericordia qui connaîtra une nouvelle édition l'année suivante, et 10.000 exemplaires de El Abuelo qui sont épuisés en 1904 (cf. document 9). Or ces deux romans ont été publiés la même année que celui d'Unamuno.

Ce qui est impressionnant, ce qui prédomine, c'est donc l'oeuvre totale, l'ensemble de l'opus (réduit ici dans le cadre de

21 Apud Rafael Pérez de la Dehesa, «Editoriales e ingresos literarios a principios del siglo» (Revista de Occidente, 1969, n. ${ }^{\circ} 71$, p. 218). Le rythme de vente de Pepita Jiménez aurait donc été de 1874 à 1904 de 111 exemplaires par mois.

D'après des calculs effectués par Jean Le Bouill, dans la longue durée (plus de 30 ans), Peñas arriba, Sotileza, El sabor de la tierruca, Don Gonzalo González de la Gonzalera. Nubes de Es"io, Al primer vuelo, La Montálvez, La Puchera, De tal palo, Hombres de pro, El Buev suelto et Pachín ont tous des rythmes de vente mensuels supérieurs à 50 exemplaires. c'est-à-dire 20 exemplaires de plus en moyenne que l'ensemble des romans de Galdós... / $C$. f. Jean Le Bouill. Les Tableaux de moeurs et les romans ruraux de José María de Pereda... Université de Bordeaux III, 1980, pp. 159-162). 
cette étude à 30 romans et 46 Episodios), qui donne à Galdós et à son oeuvre cette audience exceptionnelle pour l'Espagne de l'époque ${ }^{23}$.

3.1.2. L'audience. En effet, si on applique aux volumes de chaque titre vendus entre 1875 et 1905 le coefficient, connu, mais sans doute sous-estimé, pour le pays et pour l'époque, de $3,5^{24}$, on obtient pour cette première génération de lecteurs potentiels de Galdós un public compris entre 150.000 pour chaque titre de la Première série des Episodios nacionales (3,68\% des alphabétisés à la date de 1877), et 25.000 environ, pour un titre des Novelas españolas contemporáneas ${ }^{25}$.

Au vu de ces chiffres, on peut relativiser la supposée «popularité» de Galdós, et en particulier sa pénétration dans les couches populaires ${ }^{26}$, même si 150.000 lecteurs pour les Episodios c'est presque trois fois plus que le nombre moyen de lecteurs d'un roman par livraisons, d'après mes calculs ${ }^{27}$, et que 25.000 lecteurs, c'est cinq fois plus que le public de Juan Valera ${ }^{28}$.

23. Même les romans les moins vendus (à long terme) ont des rythmes égaux ou supérieurs à ceux des œuvres de Renan, Nietzche, Bakounine et Darwin (sauf L'Origine de l'homme), publiées et diffusées en Espagne et dans toute l'Amérique latine par Sempere y Cía. de 1902 à 1909 (cf. Pérez de la Dehesa, «La editorial Sempere en Hispano-América y en España», Revista iberoamericana, núm. 69, 1968, pp. 551-555).

24 Parce que, en Espagne, le circuit prête-main semble, d'après les témoignages dont nous disposons, plus important, et la dimension moyenne des familles plus grandes.

25 Le public de Doña Perfecta, Marianela, Gloria et La Familia de León Roch pendant la même période peut être estimé à 105.000 (avec le même multiplicateur de 3,5), ce qui met ces romans au niveau de la deuxième série des Episodios.

26 Sur ce point, voir A. Dérozier, «Le roman historique au XIX' siècle: la problématique d'un genre» (Communication au XIV ${ }^{e}$ congrès de la Société des Hispanistes Français, Nice, mars 1978).

Pour donner un ordre d'idées, ces 150.000 personnes représentent un groupe un peu plus nombreux que celui isolé par M. Martínez Cuadrado (La Burguesía conservadora (1874-1931), Madrid, Alianza, 1973, pp. 226-229) sous le nom de «bloque de poder económico».

27 Cf. J. - F. Botrel, «La novela por entregas: unidad de creación y de consumo». In: Creación y. público en la literatura española, Madrid, Castalia, 1974, p. 132.

28 Lui-même estime en effet son public à 5 ou 6.000 lecteurs et celui de Galdós à 20.000 (il pense sans doute au public des romans) (Cf. J. - F. Botrel, «Juan Valera et l'argent», Bulletin Hispanique, LXXII, n. ${ }^{\circ}$ 3-4, Julliet-Décembre 1970, p. 307). 
3.1.3. La durabilité. Mais l'audience de Galdós n'est pas limitée à cette première génération ni à ses contemporains: aux alentours de 1939 on peut estimer que, sans tenir compte du théâtre ni d'autres oeuvres éditées après la mort de Galdós, comme celles publiées par Alberto Ghiraldo chez Renacimiento, il y a eu 2.300.000 volumes de Galdós (1.750.000 Episodios et 550.000 romans) mis en circulation et vendus en Espagne et dans le monde hispanophone.

Cent ans après la parution du premier Episodio, Trafalgar, c'est, pour les seuls Episodios Nacionales, plus de 2.600.000 volumes qui sont allés ainsi au devant de lecteurs, répartis de façon presque égale avant et après la mort de Galdós (1.242.000 et 1.389 .500 ), ce qui traduit à la fois le grand succès et la durabilité de cette partie au moins de l'oeuvre de Galdós et, en tout cas, la validité du label d'un point de vue commercial et littéraire.

Cette grande audience du label Galdós se trouve encore renforcée si on ajoute à cette arithmétique d'autres facteurs non quantifiables actuellement, comme les éditions d'Amérique du Sud, les traductions et surtout l'accumulation des lectures successives d'un même livre sur plusieurs générations (même si l'usure ou la destruction y font obstacle), grâce aux bibliothèques publiques ou privées, au passage dans le circuit de l'occasion, etc., avec éventuellement une évolution et une transformation de la composition sociale du public originel.

Sans donner plus d'explications, pour l'instant, on comprend peut-être mieux le processus de constitution de ce que j'appelle le label Galdós, son image de marque classique, sa réputation assise sur un opus abondant, que l'accumulation des exemplaires dans l'espace et dans le temps rend massif; grâce, fondamentalement, à la pérennité et à l'universalité de son message, ou plus exactement, comme nous allos le voir, d'une partie de son message.

3.2. Le succès différencié de l'oeuvre de Galdós. Il l'est en effet parce que, au sein de l'opus, les rythmes de vente varient, 
dans la longue durée de 1 à 3 et plus, selon les différentes catégories de romans ${ }^{29}$.

Ainsi lorsque le rythme moyen de vente des cinq séries des Episodios nacionales est, jusqu'en 1939, de 79 exemplaires par mois, celui des 30 ou 32 romans n'est que de 27 et celui des oeuvres dramatiques insignifiant, à l'exception d'Electra.

En d'autres termes, les Episodios nacionales connaissent pendant cette période pratiquement trois fois plus de succès (d'édition) que les Novelas de la primera época et les Novelas españolas contemporáneas confondues ${ }^{30}$.

Au sein de chaque grande catégorie, il existe des différences minimes dans le cas des Episodios puisque les lère et $2 \mathrm{e}$ séries ont un rythme de 81 contre 74 aux trois autres, et beaucoup plus accentuées entre les Novelas de la primera época qui ont un rythme de 74, comparable donc à celui des Episodios de la seconde époque, et les Novelas españolas contemporáneas qui ont un rythme moyen de 20 seulement.

La relation entre les romans vendus 2 pesetas (Episodios et Novelas de la primera época), et ceux vendus 3 pesetas est donc à long terme de 1 à 3 ou 4 , avec une tendance à l'augmentation, qu'on devine déjà entre 1897 et 1904, puisque, si, entre le 4-111896 et le 27-05-1897, les premiers ont un rythme de 16 et les seconds de 8, entre le 15-01-1904 et le 7-05-1906, ces rythmes sont passés respectivement à 31 et à 6 .

A ces variations par grandes catégories, il faut ajouter celles qui existent au sein de chacun des groupes de romans.

3.2.1. Les Episodios nacionales. Rappelons d'abord que les Episodios ont été produits en deux temps (1873-1879 et 18981912) et que l'échelonnement de chaque série et même de chaque

29 Pour les raisons indiquées plus haut (paragraphe 11), l'œuvre dramatique de Galdós n'est pas prise en compte pour ces analyses.

30 Pourtant la maison Hernando, en précisant les tirages des éditions de nouveaux romans à deux pesetas ou à trois pesetas, ne vise que sur une différence de un à deux (16.000 exemplaires et 8.000 exemplaires respectivement) (apud J.-F. Botrel, "Galdós y la casa editorial...», loc. cit., p. 265). 
volume dans le temps a des conséquences évidentes sur les chiffres d'exemplaires imprimés: il est logique, d'un certain point de vue (celui de la durabilité de l'oeuvre galdosienne), qu'en 1973 on ait tiré presque quatre fois plus d'exemplaires de la première série que de la cinquième ${ }^{31}$.

3.2.1.1. La hiérarchie interne. Dans le tableau ci-dessous est précisé, par série, le nombre de milliers d'exemplaires d'Episodios vendus depuis le début, à chacune des dates indiquées. On peut y constater que cette hiérarchie, qui va de la première à la cinquième série, est confirmée, les différences étant plus accentuées entre la lère et la 2ème qu'entre les 3ème, 4ème et 5ème.

$\begin{array}{lcccc} & 1905 / 08 & 1920 & 1939 & 1973 \\ \text { lère série } & 402 & 452 & 693 & 913,5 \\ \text { 2ème série } & 331 & 377 & 516 & + \text { de } 660 \\ \text { 3ème série } & 104 & 161 & 258 & 430 \\ \text { 4ème série } & 60 & 154 & 290 & 390 \\ \text { Sème série } & & 98 & 181 & 238\end{array}$

Mais cette hiérarchie absolue, doit être nuancée par l'étude des rythmes qui montre que les variations internes ne sont pas exactement semblables.

D'après les documents 11 à 15 , on peut observer:

-dans le premier groupe un succès relatif plus important pour la série animée par Araceli que pour celle animée por Salvador Monsalud (75 contre 59).

- dans le second groupe, la 5e série, incomplète mais plus contemporaine par ses thèmes et ses dates de publication et, par là même, peut-être moins victime de l'usure, est celle qui a connu le plus de succès dans la longue durée au point qu'elle atteint presque le rythme de la lère série: 71 exemplaires par mois. La 4ème série, un peu moins contemporaine par le sujet et les dates, n'atteint que le chiffre de 60 (équivalent donc à celui de la 2ème)

31 Le phénomène d'usure peut jouer, on le sait, pour les oeuvres les plus anciennes. Dans le cas de Galdós, ce phénomène ne se traduit, cependant, pas par des effets majeurs puisque ce sont presque toutes les oeuvres antérieures à 1880 qui ont connu le plus grand succès, absolu et relatif. 
et la 3ème, qui est peut-être, avec la 1ère, la série la plus commentée par la critique, a le succès le plus faible: 53.

Les différences internes sont, on le voit, assez limitées, même si le succès de la 1ère série est de $70 \%$ supérieur à celui de la 3ème série.

Ces différences ont connu des variations conjoncturelles, générales et particulières.

3.2.1.2. Après 1939. Pour toutes les séries, par exemple, le rythme mensuel de vente est plus élevé avant qu'après 1940, avec des variations par rapport à la moyenne génerale de chaque série, de 50,99\% dans le cas de la 4ème et de $17,64 \%$ dans le cas de la lère, ainsi qu'on peut l'apprécier dans le tableau suivant.

$\begin{array}{lccc} & \begin{array}{c}\text { Rythme moyen } \\ \text { de vente } \\ \text { jusqu'en } \\ 1953\end{array} & \begin{array}{c}\text { Rythme moyen } \\ \text { de vente } \\ \text { jusqu'en } \\ 1940\end{array} & \begin{array}{c}\text { Rythme moyen } \\ \text { de vente } \\ \text { de } 1940\end{array} \\ \text { 1ère série } & 75 & 88(+17,64 \%) & 64(-14,66 \%) \\ \text { 2ème série } & 59 & 74(+26,34 \%) & 52(-11,86 \%) \\ \text { 3ème série } & 53 & 66(+24,88 \%) & 45(-15,06 \%) \\ \text { 4ème série } & 60 & 79(+50,99 \%) & 42(-30,00 \%) \\ \text { 5ème série } & 71 & 87(+24,67 \%) & 42(-40,84 \%)\end{array}$

On peut donc dire que, sous le Franquisme, le succès des Episodios nacionales édités par la Casa editorial Hernando, S.A. diminue globalement et régulièrement. Ce processus de désaffection touche davantage les 5ème et 4ème séries, et dans une moindre mesure la 3ème, que la 2ème ou la 1ère; d'ailleurs pendant 20 ans (jusqu'en 1973) la maison Hernando ne va plus faire d'éditions des Episodios de la seconde époque. Pour les éditions de 3.000 exemplaires faites en 1952-1954, cela donne un rythme de 12 exemplaires par mois!

Quant aux deux séries de la première époque, elles continuent à être éditées (42 éditions de 1955 à 1973 pour les 20 titres), mais leur rythme de vente baisse également: il passe, pour la lère série, de 64 avant 1953 à 43 après, tandis que la 2ème s'effondre de 52 à $16(-225 \%)$. 
On peut donc dire que seule vraiment la lère série résiste au Franquisme, mais à un niveau qui la ramène aux années 19041906, c'est-à-dire aux années les plus mauvaises pour elle.

Les dernières années du Franquisme auront donc été plus néfastes aux Episodios que les premières où pourtant Luis Araujo Costa exprimait, dans son prologue de 1941 à Trafalgar, toutes les réticences idéologiques du nouveau pouvoir à l'égard de Galdós et de «ses erreurs doctrinales et d'appréciation, son attitude inélégante lorsqu'il partage et propage les leurres avec lesquels l'Anti-Espagne essaie d'étouffer notre esprit et la nature même de notre être comme nation...» ${ }^{32}$.

On ne peut néanmoins ignorer l'incidence éventuelle d'autres facteurs étrangers au signe idéologique ou au message de Galdós, comme la concurrence possible des Obras completas, l'absence d'actualisation des éditions, sans notes et avec la traditionnelle couverture rouge et or, etc. On remarquera ainsi que la réédition des Episodios par la puissante Alianza editorial, avec des couvertures plus attirantes, en relancera la vogue. Mais il faut dire qu'entretemps s'est amorcé un courant de revendication de Galdós par l'Espagne officielle qui patronne le Premier congrès international d'études galdosiennes à Las Palmas en 1973 et récupère Galdós pour un libéralisme de façade.

Reste, au bout du compte, que le Franquisme n'est pas une période faste pour l'oeuvre de Galdós, même pour ses Episodios nacionales.

3.2.1.3. Avant 1939. Avant, le rythme a été supérieur à la moyenne générale. On note néanmoins une baisse générale et régulière d'intérêt qui affecte toutes les séries jusqu'en 1906-1914 et se continue au delà, sous forme de stagnation pour les 4 dernières, en épargnant la lère série.

Examinons cette évolution par sous-période et pour chaque époque d'Episodios. greso internacional de estudios galdosianos. Ediciones del Excmo. Cabildo Insular, 1977, p. 73. 


\subsection{Jusqu'en 1914 .}

3.2.1.3.1.1. lère et 2ème séries. Jusqu'en 1904-1908, le rythme de vente des lère et 2ème séries baisse régulièrement, de l'ordre de $60 \%$ en 20 ans (de 100 environ à 40 environ) avec une légère et passagère récupération de la lère série pendant le temps où Galdós est son propre éditeur.

La lère série (à l'exception de Trafalgar dont le cas sera traité à part) a un rythme élevé (212) de 1873-75 à 1874-78, succès qui, on le sait, surprit, au moins au début, l'auteur et l'éditeur ${ }^{33}$. Le rythme baisse ensuite de moitié (100) jusqu'en 1884-86, et se maintient à ce niveau jusqu'en 1887-1890, approximativement. Il se produit ensuite une nouvelle chute importante puisque le rythme n'est plus que de 55 entre 1887-90 et 1896-97. La légère reprise qu'on enregistre avec la maison d'édition Obras de Pérez Galdós n'est cependant que passagère, puisque, sous la gestion d'Hernando, le rythme a encore baissé (40 en 1904-06) et se maintient à 49 jusqu'à la veille de la Première guerre mondiale.

Quant à la 2ème série, elle se maintient à un rythme d'un peu plus de 100 exemplaires de 1877-79 à 1884-1886, soit presque moitié moins longtemps que la 1ère série dont le rythme moyen est de 107 de 1873-75 à 1884-86. Pourtant l'usure de la 2ème série est moindre et elle bénéficie du succès de la lère dont 128.000 exemplaires environ ont été vendus en 10 ans.

Entre $1884-86$ et $1892-93$, le rythme de la 2ème série se situe aux environs de 83 et il baisse encore ensuite, en se maintenant cependant à un niveau légèrement supérieur à celui de la lère série dans les années immédiatement antérieures à la création de la maison d'édition Obras de Pérez Galdós (66 de 1892-93 à 18971900 ). Mais, en 1904, son rythme se trouve à nouveau en dessous de celui de la lère (31 exemplaires) et, de 1904 à 1908, cette situation s'améliore à peine.

33 Sur ce point, voir Jacques Beyrie, op. cit., p. 168. 
Pendant cette première période, les deux premières séries connaissent donc une évolution semblable, mais la lère série semble, d'ores et déjà, jouir d'une acceptation plus grande.

3.2.1.3.1.2. 3ème, 4ème et 5ème séries. Les Episodios de la seconde époque, dont la publication commence 25 ans environ après celle de la première série, sont, dans leur évolution jusqu'en 1914, caractérisés par les faits suivants:

1. Des premières éditions d'un nombre d'exemplaires beaucoup plus important que pour les deux premières séries, comme si Galdós voulait àpprovisionner immédiatement le marché qu'il s'est constitué avec les deux premières séries et qu'il a eu le temps de repérer.

2. Un rythme de vente initial bien supérieur également à ceux des lère et 2ème séries: 126 de 1898 à 1906 pour la 3ème série ${ }^{34}$, entre 250 et 166 pour les cinq premiers titres de la 4 ème.

Ce rythme est bien sûr beaucoup plus élevé durant les premiers mois: 394 d'avril 1904 à mai 1906 pour La Revolución de Julio, 618 de juin 1904 à mai 1906 pour O'Donnell, 618 pour Aita-Tettauen depuis février 1905 et 747 pour Carlos VI en la Rápita de juin 1905 à mai 1906, ce qui donne, pour ce dernier titre, près de 9.000 exemplaires écoulés en un an.

3. Cependant ce rythme de vente chute brusquement. Ainsi, par exemple, la 3ème série passe de 126 entre 1898 et 1906 à 33 entre 1904 et 1906, c'est-à-dire à un rythme très inférieur à celui des deux premières séries à ces dates, et si elle semble récupérer peu après (59 en 1906-1909), la moyenne 1904-1909 reste très basse ${ }^{43}$, ce qui représente en 10 ans une baisse de $66 \%$ alors que les deux premières séries ont mis 20 ans pour baisser de $60 \%$.

De succès immédiat de la 3ème série est souligné par F. C. Sáinz de Robles dans son Introduction aux Obras Completas de Galdós (Madrid, Aguilar, 1950, t. I, p. 81): en deux ans, écrit-il, on a vendu plus de 50.000 exemplaires des 10 tomes, quantité invraisemblable pour ce qu'était, dans l'Espagne de cette époque, la demande en lecture. Si nous appliquons le rythme calculé de 126 à ces deux ans et aux 10 tomes, on trouve plus de 30.000 exemplaires vendus ce qui, étant donné que notre rythme est calculé sur 7 ans rend tout à fait vraisemblable le chiffre indiqué par Sáinz de Robles. 
Tout se passe donc comme si, avec les 12 ou 14.000 exemplaires de la première édition de chaque titre, nous avions la totalité de la clientèle immédiate des nouveaux Episodios dans les années 1900 et que les nombreux exemplaires imprimés durant la première année saturaient rapidement le marché.

On observe, d'autre part, que si les exemplaires du premier tirage de 8.000 ou 5.000 de la 3ème série ne mettent que deux ans, à peu près, à être épuisés, les 4.000 suivants mettent cinq ans et les 4.000 ultérieurs plus de 20 ans ( $c f$. document 4). Cette évolution générale est d'ailleurs confirmée par l'espacement des éditions au fur et à mesure que les dernières séries sortent: 1898, $1900,1906,1924$ pour la 3ème, 1902, 1907, 1926 pour la 4ème et 1912, 1929 pour la 5ème ( $c f$. document 7) ${ }^{35}$, Les 12.000 exemplaires de la première édition de Narváez (4ème série) mettent quatre ans à s'épuiser alors qu'il faut cinq ans a Los Duendes de la Camarilla et à La Revolución de Julio (4ème série) pour épuiser le même nombre d'exemplaires. Quant aux 16.000 exemplaires de De Cartago a Sagunto (5ème série), ils ne seront épuisés que 13 ans après, puisque ce n'est qu'en 1924-25 qu'on fait une nouvelle édition de 2.000 exemplaires. Les 20.000 exemplaires de la première édition très optimiste de Cánovas (1912) mettront, eux, 17 ans à se vendre.

On dirait donc que la reprise de la publication des Episodios nacionales en 1898 ne permet pas de retrouver un public équivalent à celui des deux premières séries et si le succès des dernières séries est, au début, apparemment plus fulgurant que celui des deux premières, la pression de la demande baisse rapidement et ne se réveille pas par la suite.

Qui sont ces 10 ou 12.000 acquéreurs enthousiastes des nouveaux Episodios? Un dernier carré de fidèles des deux premières séries ou une nouvelle génération qui n'adhère guère à un genre déjà vieilli, même si dans une certaine mesure la perspective de

35 Dans le document $n .^{\circ} 7$, on peut percevoir, visuellement, les différentes politiques d'édition: pour les deux premières séries, les éditions sont rapprochées et régulières jusqu'en 1970, tandis que pour les trois dernières les points noirs représentatifs des éditions sont espacés, de plus en plus espacés jusqu'à leur disparition en 1953 . Ver nota 4. 
Galdós a changé? Sont-ils les victimes de la marque Galdós, d'une marque connue, en particulier de la presse? Ce qui est sûr, c'est qu'aucune des trois dernières séries ne réussit à se maintenir dans le moyen et le long terme au niveau des deux premières et que la tendance à l'obsolescence est d'autant plus rapide que la série est plus contemporaine (historiquement) et plus avancée dans le siècle: la dernière série est de ce point de vue la plus durement touchée ${ }^{36}$.

3.2.1.3.1.3. La chute de 1904-1906. Reste à expliquer la brusque chute survenue en 1904-1906 qui a certainement à voir avec la marche des affaires de la maison d'édition Obras de Pérez Galdós et les bouleversements occasionnés par la transmission de la gestion des stocks et de la responsabilité éditoriale (du moins en partie) à Perlado, Páez y Cía. ${ }^{37}$.

Pendant ces deux années de vaches maigres, se font sentir de façon plus marquée des tendances qui ensuite seront confirmées, comme, par exemple, la position de la 2ème série par rapport à la 1ère et celle de la 3ème (rythme de 33) qui, quatre années seulement après la publication du dernier titre (La de los tristes destinos), a presque déjà le rythme de la 2ème. Pour la 4ème série, on ne dispose de données que pour Las tormentas del 48 et Los duendes de la camarilla qui tous deux ont un rythme de 44, logiquement supérieur à celui de la lère série qui nous sert de référence, étant donné la proximité de leur parution (1902 et 1903), mais néanmoins peu soutenu.

Dans les années suivantes, on note une certaine reprise, de faible importance toutefois.

C'est pourquoi on peut considérer les années 1904-1906 qui suivent immédiatement l'échec de l'expérience Obras de Pérez Galdós mais aussi le «scandale» d'Electra, comme une phase ré-

36 Le rythme de La Primera República est de 102 de 1911 à 1924 et de 23 de 1924 à 1935 , celui de De Cartago a Sagunto de 105, de 1911 à 1931, et celui de Cánovas de 98, de 1912 à 1929, et 56, de 1929 à 1935 ( 83 de 1912 à 1935).

37 Cette chute affecte également les romans qui ont alors un rythme six fois moins élevé que précédemment, tout en conservant la hiérarchie interne ( $C f$. document $\left.n .^{\circ} 17\right)$. Ver nota 4 . 
vélatrice d'une évolution décisive dans le comportement de l'oeuvre de Galdós: une perte de clientèle et d'acceptation générale que l'on note à propos de 3ème, 4ème et 5ème séries des Episodios. Cette phase coïncide d'ailleurs avec une période de création plus orientée vers le théâtre (El abuelo (1904), Bárbara (1905), Amor y ciencia (1905), qui ne remportent guère de succès d'ailleurs) que vers le roman: après Casandra, publié en 1905, il faudra attendre jusqu'en 1909 pour voir publié El caballero encantado, puis 1915 pour La Razón de la sinrazón, même si Galdós poursuit la publication de la 4ème série d'Episodios.

3.2.1.3.2. De 1914 à 1931. À partir de 1914, les chemins de la lère et de la 2ème série divergent.

3.2.1.3.2.1. La première série des $E$. $N$. Curieusement le rythme de ventes de la lère série va en augmentant (79 de 1914 à 1929) jusqu'à atteindre, dans les années 1929-1939 un rythme semblable à celui des années 1873-1886: 105.

Cette croissance (forte par rapport au creux de 1904-1914) s'accompagne bien sûr d'un rythme d'édition plus soutenu (éditions de 4.000 exemplaires en 1912 et 1916) dont les tirages augmentent tant entre 1914-1917 et 1928-1929 qu'au total, ce sont 93.000 exemplaires qui sont vendus (près de 10.000 exemplaires par titres) avec un rythme mensuel de 221. Cette accélération du succès d'édition est confirmée par le niveau des tirages de 19281929 , supérieurs à 5.500 exemplaires en moyenne, et par le comportement de cette série sous la République.

3.2.1.3.2.2. La 2ème série des $E$. $N$. La rythme de vente de la 2ème série, appréciable uniquement à travers celle de deux titres (Siete de Julio et El terror de 1824 - mais nous verrons que le succès de chaque titre est peu différent de celui de l'ensemble de chaque série) est, entre 1907 et 1939, inférieur à la moyenne générale ${ }^{38}$ : aux alentours de 55 ou moins, soit à peu près au niveau de 1906-1908 avec un rythme d'édition régulier mais avec des tirages limités.

38 Voir dans le document n. ${ }^{\circ} 9$ les chiffres des éditions de 1906 à 1934 et, par déduction, le nombre d'exemplaires vendus: environ 15.000 pour El terror de 1824 et 19.000 pour Siete de Julio. 
3.2.1.3.2.3. La 3ème série des $E$. N. Par rapport à 19041909, le rythme de la 3ème série baisse: 36 pour Mendizábal entre 1906 et 1934; il est désormais inférieur à celui de la 2ème série malgré une certaine reprise entre 1922-1925 et 1929-1930, attestée par les éditions faites à ces dernières dates et confirmée sous la Seconde République.

3.2.1.3.2.4. 4ème série des $E$. $N$. Le rythme de la 4ème série suit la tendance signalée pour la 2ème et la 3ème à la stagnation puisque de 1906-1909 à 1929-1931 ne sont vendus que 11 à 13.000 exemplaires de chaque titre avec un rythme de 39 ou 40 entre 1906-1909 et 1925-1926 (dates d'une nouvelle édition) qui augmente un peu par la suite puisque le rythme entre 1906-1909 et 1929-1931 varie entre 42 et 50 selon les titres, ce qui le met un tout petit peu en dessous du rythme de 1904-1909.

3.2.1.3.2.5. 5ème série des $E$. $N$. Les calculs réalisables pour la Sème série dont on fait des éditions en 1924-1929 et 19311935 confirment cette stagnation bien qu'à un niveau nettement supérieur: le rythme de De Cartago a Sagunto est de 83 entre 1912 et 1935 (98 de 1912 à 1929) et celui de Cánovas est de 95 de 1911 à 1925 et de 97 de 1925 à 1931.

3.2.1.3.3. Sous la Seconde République. C'est sous la République qu'apparaissent les fameuses couvertures tricolores (les trois couleurs du drapeau républicain) dont on imprime 60.000 exemplaires le 4 février 1933, et qui sont apparemment épuisées 26 mois plus tard, puisqu'en mai 1935 on en fait un nouveau tirage de $40.000^{39}$. Cela donnerait pour l'ensemble des Episodios nacionales (et non pour chaque titre) un rythme de vente de 2.300 exemplaires par mois entre 1932 et 1935.

Entre 1931 et 1939 , on réédite ( $c f$. document n. $\left.{ }^{\circ} 7\right) 27$ titres sur les 46 que comprennent les $\mathrm{E}$. $\mathrm{N}$.

De la lère série, Hernando imprime 4.000 exemplaires de chaque titre (sauf de La Corte de Carlos IV), avec deux éditions sup-

39 Selon le Registro de trabajos entregados a la imprenta. Libro II. 1-09-1930 - 4-04-1939, les 60.000 couvertures imprimées correspondent exactement au nombre d'exemplaires tirés par Hernando de février 1933 à mai 1935 ( $c f$. document n..$^{\circ}$ ). 

1939, est faite une nouvelle édition de sept titres, sans précision de tirage ${ }^{41}$.

\title{
Dans la 2ème série, on trouve une édition de 4.000 exemplai-
} res de El equipaje del Rey José et de Siete de Julio et une de 6.000 pour Memorias de un cortesano et Los cien mil hijos de San Luis.

\author{
Les quatre titres de la 3ème série réédités sont: Mendizábal \\ (6.000 exemplaires), La Campaña del Maestrazgo (4.000), Zuma- \\ lacárregui et Los Ayacuchos (sans précision de tirage).
}

40 Le cas de Trafalgar est un peu exceptionnel dans la mesure où il s'agit du premier tome des Episodios nacionales, le livre qu'on achète et lit, même si on abandonne par la suite la série, mais aussi un livre qu'il faut avoir lu, classique, en raison de l'événement dont il traite, une défaite dont retentissent les leçons d'histoire de l'enseignement primaire. Ces facteurs conjugués font que Trafalgar a un rythme de vente général plus élevé, de $23 \%$, que la moyenne de la 1ère série ( $18 \%$ seulement jusqu'en 1939 ), avec une tendance à l'accentuation de cette différence à partir de 1914 ( 71 entre 1907 et 1914; 129 entre 1914 et 1939) mais aussi les mêmes creux que l'ensemble de la série (104 entre 1904 et 1909, 67 de 1941 à 1971).

Les tentatives pour isoler le comportement éditorial d'autres titres des E. N. s'avèrent assez décevantes. Avec beaucoup de précautions, on pourrait peut-être dire qu'après 1940, Zarago$z a$ atteint pratiquement le niveau de Trafalgar (67), avant et surtout après 1953, même en nombre d'exemplaires. Mais avec un message similaire (résistance de l'Espagne assiégée, valeurs héroïques, sentiment anti-français), Gerona n'a pas un comportement remarquable.

Dans la 4ème série, on remarque aussi un comportement légèrement différent de La de los tristes destinos et de Prim dont les rythmes sont supérieurs à la moyenne de la série (76 et 69 respectivement contre 60), cette supériorité étant plus affirmée avant 1930-1940 (86 et 97/60) qu'après. Y aurait-il un engouement particulier pour ces titres? Dans la Sème série c'est $D e$ Cartago a Sagunto qui semble le plus acheté: 105 contre 87 à l'ensemble de la série jusqu'en 1931-1935. Y-a-t-il un lien entre la thématique de ces titres et leur succès un peu plus accentué? Il faudrait trouver des confirmations en dehors des chiffres... Car force est de constater qu'en règle générale, les lecteurs ne semblent pas avoir isolé les titres de la série qui est conçue et considérée comme un roman à épisodes. Bien sûr on trouvera -et c'est logique- des rythmes de vente un peu plus élevés pour les premiers titres (ainsi de 1873 à 1971 La Corte de Carlos $I V$, deuxième titre de la lère série, a un rythme de 76 et La batalla de los Arapiles (dernier de la série) un rythme de 66, et dans la 2ème série El equipaje del Rey José est à 62 quand Un faccioso más... est à 58 . Il y a toujours des lecteurs à abandonner la série en cours de route, mais on remarquera que, en tout état de cause, ces différences sont minimes, ce qui confirme l'attitude et la pratique du public (des acquéreurs) à l'égard de séries d'Episodios considérées comme un tout. Après 1940, d'ailleurs, les tirages se font pour tous les titres, à la même date et au même niveau, ce qui suggère des achats en bloc (pour des bibliothèques par exemple).

41 Entre 1936 et 1939 on remarque une diminution du rythme qui devient, sauf pour Trafalgar, Cádiz et Juan Martín el Empecinado, inférieur à la moyenne jusqu'en 1939. 
Il semble qu'on traite différemment les 4ème et 5ème séries puisque, de la 4ème, on réédite à 6.000 exemplaires quatre titres (La Revolución de Julio, O'Donnell, Carlos VI en La Rápita et $L a$ de los tristes destinos) et, de la 5ème, les six titres.

Ces séries un peu oubliées durant la période antérieure (surtout la 4ème) semblent mériter à nouveau, sous la République, un certain intérêt. L'épuisement des éditions précédentes coïncide, il est vrai, avec la République, mais la niveau des tirages (6.000 exemplaires) semble indiquer que l'éditeur prévoit un élargissement du marché pour ces séries aussi.

On ne peut cependant dire que le rythme général des $\mathrm{E}$. N. sous la République soit trés élevé: la 2ème série, pour les titres accessibles, se trouve à peu près au niveau moyen pour 1875-1930 (71 et 83/74) et la 3ème presque au niveau de 1898-1936. Cela suppose néanmoins une bonne récupération par rapport à la période immédiatement antérieure.

Il faut aussi considérer à part la lère série dont le rythme de vente augmente énormément: 145 entre 1928-1929 et 1934-1939 alors que la moyenne entre 1873 et 1939 est de 88 , soit plus $65 \%$. Dans cette série Trafalgar atteint le rythme record de 266 entre octobre 1934 et janvier 1936 (125 entre août 1932 et octobre 1934 , bien au dessus de sa moyenne particulière entre 1873 et 1971 (92) et entre 1873 et 1939 (107) qui se situe déjà bien au dessus de celle de tous les autres titres de Galdós ( $c f$. document $n .^{\circ}$ 19).

Les rééditions commandées par le Gouvernement espagnol en 1939, en plein siège de Madrid, à une imprimerie sous séquestre (incautada), confirment que de tous les Episodios nacionales, ce sont ceux ayant trait à la Guerre d'Indépendance qui connurent, par leur actualité, le plus de succès; ils furent, comme le rappelait Rafael Alberti en 1943, "reçus par les soldats, à côté du fusil, avec une faim semblable à celle de pain dans les tranchées, désirés comme un renfort attendu dans l'épuisante bataille»" ${ }^{42}$.

42 «Un episodio nacional: Gerona», reproduit dans El escritor y la crítica, Benito Pérez Galdós, Edición de Douglass M. Rogers, Madrid, Taurus, 1973, p. 370. 
3.2.1.4. Le succès des Episodios nacionales de 1873 à 1973. Le comportement éditorial des Episodios nacionales de 1873 à 1973 est, donc, caractérisé par:

1. Une tendance à la baisse de succès des cinq séries jusqu'en 1904-1914, qui sont des années de creux accentué, et à une baisse beaucoup plus rapide dans le cas des 3ème, 4ème et 5ème séries.

2. Une stagnation des 4 dernières séries après 1914-1920 et une croissance de la lère jusqu'à retrouver son niveau des premières années.

3. Une réactualisation sous la République, particulièrement sensible pour la 1ère série pendant la Guerre civile, et un renversement total de la tendance sous le Franquisme, avec un déclin général qui affecte beaucoup moins la lère série ( $c f$. document 20).

3.2.2. Le succès des romans ${ }^{43}$. On a l'habitude à des fins bibliographiques de distinguer dans l'oeuvre romanesque de Galdós les Novelas de la primera época (N. P. E.) et les Novelas españolas contemporáneas (N. E. C.). Certains spécialistes de Galdós (Casalduero, Montesinos, ou Sáinz de Robles) ont proposé ou établi d'autres critères, mais il faut bien constater que cette classification suggérée par Galdós lui-même ou son éditeur, correspond, du point de vue du succès d'édition, à une nette différenciation. Il faut simplement mettre à part, dans les Novelas de la primera época, les cas des trois premiers romans (La Fontana de Oro, El Audaz, La Sombra) qui font partie de la «période historique» définie par Joaquín Casalduero et sur le comportement éditorial desquels on dispose de peu d'informations ${ }^{44}$.

3.2.2.1. Hiérarchie interne. On remarque, en effet, une nette différence dans les niveaux et dans la durée du succès entre Do-

43 L'étude des variations conjoncturelles du succès des romans est encore moins facile que dans le cas des E. N., en particulier pour la première réception. Sous le Franquisme, le fait que la maison Hernando ait cédé les droits de reproduction de la plupart des Novelas españolas contemporáneas à d'autres maisons d'édition fait que l'étude ne peut porter que sur quelques titres.

44 Celles qui existent ont été exploitées par Jacques Beyrie, op. cit., p. 73 et 59. 
ña Perfecta, Gloria, Marianela et même La familia de León Roch (rythmes au dessus de 40) et tous les autres romans espagnols contemporains (NEC) dont les rythmes de vente sont compris, jusqu'en 1936, entre 17 et 27.

Le document $n .^{\circ} 16$ traduit cette différence, dans la longue durée, qui va de 1 à 6 entre Marianela et Tristana (cas extrême) mais est, en général, de l'ordre de 1 à 3 ou 4 entre Marianela, Gloria et Doña Perfecta et les romans postérieurs à 1880-1881. Pour citer quelques cas: Marianela a eu, jusqu'en 1936, un rythme de vente quatre fois plus élevé que El Doctor Centeno, Miau ou Torquemada en la hoguera; Gloria a le même avantage sur Torquemada y San Pedro, et Doña Perfecta a été trois fois plus vendu que El amigo Manso ou Nazarín ${ }^{45}$.

Cette différence semble s'accentuer au fil des années puisque, en 1904-1906, ces différences étaient moindres: l'écart maximum est alors de 1 à 3 et quelque entre Doña Perfecta et El amigo Manso et Nazarín et l'écart moyen de 2 à 2,5; ainsi Gloria est pendant ces deux ans vendue deux fois plus seulement que Realidad et Marianela à peine deux fois plus que Torquemada en el Purgatorio ( $c f$. document $n .^{\circ} 17$ ). Ces variations dans la hiérarchie «définitive» sont accompagnées au sein même des NEC par une accentuation des écarts au fil des années.

Il faut remarquer que, à cette époque, on peut déjà apprécier le caractère de «roman de transition" que Donald L. Shaw atribue à La Familia de León Roch ${ }^{46}$ puisque, bien qu'étant de la même veine que Gloria ou Doña Perfecta, il annonce une rupture qui se produira, en 1881 , avec La Desheredada du point de vue esthétique mais aussi éditorial: par rapport à Marianela, Gloria et Doña Perfecta, il a un rythme de vente à peu près deux fois moins élevé: 35 contre 84 en 1904-1906 et 43 contre respectivement 99,82 et 72 jusqu'en 1936. La bibliométrie confirme donc ce «point critique» ${ }^{47}$.

45. On remarquera que cette ligne de partage coïncide avec une différence de prix entre les romans à 2 ptas. et ceux à 3 ptas.

46 Historia de la literatura española. El siglo XIX. Barcelona: Ariel, 1972, p. 205.

47 Ibid. 
La politique éditoriale suivie à l'égard des NPE et des NEC traduit bien les disparités existantes entre les deux grandes catégories de romans: tandis que, pour les romans publiés entre 1876 et 1879 , les éditions se succèdent rapidement, le rythme se relâche entre La Desheredada et Tormento, même si l'on remarque une certaine densité d'éditions jusqu'en 1893 ( $c f$. document $n .^{\circ}{ }^{8}$ ). Après il se passera respectivement $19,17,17$ et 18 ans avant qu'on ne fasse de nouvelle édition de La Desheredada, El Amigo Manso, El doctor Centeno et Tormento.

A partir de La de Bringas, la première édition n'est pas suivie d'une autre à court terme: cela veut dire que, sauf pour Torquemada en la Hoguera et Nazarín, dont on fait une nouvelle édition 9 et 12 ans après la première, il faut attendre 13 ans en moyenne avant de voir une nouvelle édition des romans postérieurs à Tormento (15 pour El Abuelo et 29 pour Fortunata y Jacinta!).

Ce phénomène s'explique-t-il par un tirage plus élevé de la première édition? Les données disponibles ne permettent pas d'envisager l'hypothèse de tirages de beaucoup supérieurs à 5 ou 6.000 exemplaires. D'autre part l'évolution ultérieure des NEC semble confirmer cette tendance à l'obsolescence.

3.2.2.2. Usure et obsolescence des NEC. En effet, si pendant la période 1897-1904 la plupart des romans espagnols contemporains accessibles ${ }^{48}$ ont, ainsi que La Fontana de Oro, des rythmes supérieurs à leur moyenne générale ( $c f$. document 17$)$, la période qui suit leur est beaucoup moins favorable: La Fontana de Oro a un rythme de 36 de 1870 à 1931 mais de 33 seulement si l'on prolonge la période de calcul jusqu'en 1936; pour Fortunata y Jacinta ces rythmes sont de 33, de 1886 à 1916 , de 23 , de 1886 à 1929 , et de 22 , de 1886 à 1932 , avec une baisse constante donc. On constate le même phénomène pour Miau (44 de 1887 à 1907, 25 de 1887 à 1932, 12 de 1907 à 1935), Lo prohibido (24 de 1885 à 1906; 22 de 1885 à 1935) et Realidad ( 25 de 1889 à 1916; 23 de 1889 à 1936).

48 C'est-à-dire El amigo Manso, Fortunata y Jacinta, Miau, Torquemada en la hoguera, Realidad, Angel Guerra, Tristana, Torquemada en la cruz, Torquemada en el Purgatorio, Torquemada y San Pedro, Nazarín et Halma. 
Cette tendance générale des NEC est perceptible dans les oeuvres en plusieurs tomes, les derniers ayant un rythme de vente supérieur à celui des premiers. On peut le constater entre 1897 et 1904 à propos de Fortunata y Jacinta où pourtant 17 mois seulement séparent la lère partie de la 4ème: celle-ci a un rythme de 32 , la 3ème de 31 , la 2ème de 28 et la lère de 27 . Le même phénomène affecte Angel Guerra dont la troisième partie, publiée 12 mois après la première, a un rythme de 33 contre 27 à celle-ci et 30 à la 2ème. Il s'agit, dira-t-on, de différences minimes sujettes à caution pour les raisons indiquées dans l'introduction. La constance de cette tendance peut néanmoins nous suggérer un phénomène d'usure du roman dont le premier tome est progressivement moins acheté, les premiers acquéreurs poursuivant jusqu'à la fin leur lecture du roman fragmenté en volumes.

La plupart des Novelas españolas contemporáneas accessibles $^{49}$ restent donc à un niveau léthargique très proche de la mort, après celle de leur auteur survenue en 1920 mais aussi, dans bien des cas, avant, alors que Gloria au Marianela retrouvent un rythme plus élevé après 1906.

D'autres romans comme La Desheredada, Tormento, La de Bringas, Lo prohibido ou Miau survivent tant bien que mal et ont même des rééditions en 1932 et 1935 ; leur rythme de vente cependant n'est jusqu'à ces dates que de 12 en moyenne, si l'on se base sur une édition de 4.000 exemplaires en 1906.

La Familia de León Roch n'échappe pas non plus à ce courant de désaffection, puisque sa dernière édition en 1939 est celle de 1920 précisément, avec un total d'exemplaires publiés de 30.000 .

Cette usure de la majeure partie (certains diront de la partie majeure) de l'oeuvre romanesque de Galdós peut étonner, même si l'on a dans la mémoire des déclarations très défavorables à son oeuvre dès le début du XXe siècle et qu'on sait que les oeuvres lit- 
téraires ont un taux de mortalité particulièrement élevé. Cela permet de constater, une fois de plus, que l'importance historique et littéraire d'une oeuvre n'est pas directement fonction de son succès d'édition immédiat ni même ultérieur et de tempérer l'enthousiasme quantitativiste des bibliométreurs.

3.2.2.3. Les oeuvres atypiques. Il existe cependant dans l'oeuvre romanesque de Galdós quelques oeuvres qui ont un comportement atypique et qui connaissent donc un succès d'édition prolongé.

C'est d'abord le cas de Marianela dont le rythme de vente croît de façon continue: 76 de 1878 à 1914, 83 de 1878 à 1917, 99 de 1878 à 1932. C'est aussi celui de Gloria (76 de 1877 à 1904; 82 de 1877 à 1935), et, de façon peut être plus inattendue, mais à des niveaux nettement inférieurs, de El Abuelo (35 de 1897 à 1928 et 38 de 1897 à $1936^{50}$ et de Misericordia (35 de 1897 à 1928; 38 de 1897 à 1936; 54 de février 1932 à janvier 1936 et 59 de février 1945 à mars 1972). Comme Doña Perfecta (pour laquelle on manque de données interprétables), ces quatre romans sont réédités à intervalle régulier ( $c f$. document $\left.n .^{\circ} 8\right)$.

3.2.2.4. Sous la Deuxième République. Avec l'avènement de la République, ce courant d'intérêt tout relatif pour l'oeuvre romanesque de Galdós se réveille un peu: douze romans bénéficient, entre 1931 et 1939, de nouveaux tirages: Gloria et Marianela (4.000 exemplaires en 1935 et 1932 respectivement) et surtout -ce qui du point de vue de l'histoire littéraire est plus intéressant-, les premiers romans espagnols contemporains, ceux de la période «naturaliste» comme La Desheredada (1932-1933), Tormento (1933), La de Bringas (1933), Lo prohibido (1935), Miau (1935) dont les dernières éditions remontaient à 1906.

Hernando réédite également Angel Guerra (1936) et Nazarín (1933), mais avec une très grande prudence puisque ces éditions, comme celles des romans précédents, ne sont que de 2.000 exemplaires.

50 Ce rythme est de 43 en 1904-1906 en raison de la proximité de la parution et peut-être auıssi de l'adaptation théâtrale. 
Seul Misericordia connaît pendant cette période un net regain d'intérêt: avec deux éditions de 2.000 exemplaires en 1932 et 1936 , son rythme de vente passe de 30 à 54 , quand, depuis 1897 , 12.000 exemplaires seulement avaient été mis en vente et qu'aucun nouveau tirage n'avait été fait depuis cette date ${ }^{51}$.

3.2.2.5. Sous le Franquisme. Sous le Franquisme, l'étude du comportement éditorial des romans est plus fragmentaire puisqu'Hernando ne continue à éditer que neuf romans ${ }^{52}$. Elle ne manque cependant pas d'intérêt.

Ainsi on devra se demander pourquoi on «ressuscite» Nazarín, 36 ans après sa dernière édition de 2.000 exemplaires en 1933, en faisant un tirage de 5.033 exemplaires en décembre 1969? Pourquoi tire-t-on soudain d'Angel Guerra 9.168 esemplaires brochés et 2.136 cartonnés en juillet 1972 quand, depuis les 2.000 exemplaires de 1936, aucun nouveau tirage n'avait été fait? Interviennent ici, naturellement, des facteurs extralittéraires tels que le cinéma ou la télévision qui viennent réveiller une demande quasiment inexistante ou du moins latente depuis plus de 30 ans ${ }^{53}$.

Il faut également remarquer les 15.000 exemplaires de Fortunata y Jacinta vendus de 1944 à 1971, date à laquelle est faite une nouvelle édition en un seul volume de 6.344 exemplaires; pendant

51 En 1932, la 4ème partie de Fortunata y Jacinta est également rééditée, probablement comme complément à l'édition de 1929. Mais il est étrange qu'aucune nouvelle édition de Doña Perfecta n'ait apparemment été faite pendant cette période, alors que le dernier tirage datait de 1927.

52 On remarquera, d'après la Bibliografía... de Manuel Hernández Suárez que c'est précisément de ces romans «léthargiques» que la maison Hernando a cédé les droits de reproduction.

Les éditions faites par Aguilar dans la Colección Crisol ne commencent qu'après 1950 et si certains romans sont réédités à plusieurs reprises, ce n'est qu'avec l'édition en livre de poche par Alianza editorial de La Desheredada (1967) puiis de Tormento (1968) qu'un certain regain d'intérêt pour ces romans de Galdós se fait véritablement sentir.

53 Ver segunda parte de este estudio. La rédition de El Doctor Centeno en décembre 1942 (4.000 exemplaires de chacun des deux tomes) est sans doute dû à ce que le roman n'avait pas été réédité sous la République C'est en tout cas une exception à la politique éditoriale d'Hernando qui ne craint pas, en l'occasion, de porter sur la couverture les invraisemblables chiffres de 100.001 et 100.003 (sic).

De El Abuelo sont vendus entre 1940 et 19725.000 exemplaires (en avril 1972, un nouveau tirage de 3.000 est fait). Cè rythme de vente modeste (13) témoigne néanmoins d'un courant d'intérêt constant. 
cette période son rythme de vente atteint 46 et est deux fois plus élevé que celui de $1886-1932{ }^{54}$. On voit, en particulier, comment l'adaptation cinématographique du roman par Angelino Fons, projetée pour la première fois au début de 1970 fait monter les ventes en flèche: 167 par mois entre 1968 et 1971.

Les 19.000 exemplaires de Misericordia vendus d'avril 1945 à mars 1972 (soit un rythme de 59, quand de 1897 à 1930 il n'avait été que de 30) confirment son succès croissant et sa reconnaissance comme «clasique». Quant à La de Bringas, elle connaît un rythme de 41 de 1952 à 1970 et de 168 de 1970 à 1972 , date à laquelle Hernando fait un nouveau tirage de 5.097 exemplaires ${ }^{55}$.

Dans tous ces cas, il faudra probablement rechercher comme pour Fortunata y Jacinta, le déclic ou la mesure qui explique le comportement atypique de ces romans.

Car pendant cette période, Gloria, dont no sont vendus que 6.700 exemplaires de 1948 à 1972, a un rythme de 23, presque quatre fois inférieur à celui d'avant 1939 (82), comme si, avec l'avènement de Franco, son message avait cessé d'intéresser ${ }^{56}$ et Marianela se trouve également bien en dessous du rythme antérieur (52 de 1942 à 1972 contre 99 de 1878 à 1932) avec en plus une tendance constante à la baisse ( 54 de 1940 à 1950 et 47 de 1954 à 1972).

Curieusement le roman de Galdós le plus publié de 1940 à 1973 semble avoir été Doña Perfecta: 28.432 exemplaires de mai 1942 à 1970, soit 4.000 exemplaires de plus que Trafalgar pour une période moins longue. Cela donne un rythme de 85 , avec une tendance à l'augmentation (54 de 1942 à 1956 et 95 de 1956 à

54 Ce rythme est de 22 . Entre 1944 et 1952, il est de 31 . A ces chiffres il faudrait ajouter les tirages, non connus, des six éditions faites en Argentine, entre 1942 et 1953, par Losada et Espasa Calpe.

55 Un nouveau tirage de 8.000 exemplaires est fait en 1972. A ces chiffres il faut ajouter les quatre éditions faites en Argentine par Losada entre 1939 et 1955.

56 La première réédition de Gloria sous le Franquisme n'a lieu qu'en 1948: les 4.000 exemplaires imprimés en juin 1936 auraient donc mis douze ans à se vendre (rythme de 28). Ne faut-il pas plutôt penser à des problèmes de censure? 
1970). Comment expliquer ce traitement différent fait à Gloria et à Doña Perfecta sous le Franquisme? Est-ce un problème de message ou bien plutôt de programmes scolaires ou universitaires? Quoi qu'il en soit, pour une œuvre réputée classique, on ne peut dire que la consommation ait été particulièrement forte tout au long de ces presque 30 ans.

3.2.2.6. Caractéristiques du succès des romans de Galdós. A propos des Novelas de la primera época et des Novelas españolas contemporáneas, on peut donc remarquer:

1. Une différence très marquée, de l'ordre de 1 à 3 ou 4 , entre le succès global de Doña Perfecta, Gloria et Marianela et celui de tous les autres romans.

2. Une tendance à l'obsolescence de plus en plus rapide et accentuée pour les Novelas españolas contemporáneas, certaines d'entre elles étant dans un état léthargique après et, dans beaucoup de cas, dès avant la mort de leur auteur.

3. Le succès relatif croissant de Marianela au fil des ans et la spectaculaire progression de Misericordia après la République.

4. La stabilité et la permanence des tendances avec une légère modification dans le sens positif sous la Deuxième République et un sens plutôt négatif (à l'exception de Doña Perfecta) sous le Franquisme ${ }^{57}$.

3.2.3. Les ouvres pour le théâtre. Les chiffres de tirage ne sont pas, on le sait, les meilleurs indicateurs de succès d'une

57 Pour la période franquiste, il faut cependant tenir compte des nombreuses éditions faites en Argentine par Losada ou Espasa Calpe à partir de 1939 et qui témoignent de la permanence d'un certain marché pour les Novelas españolas contemporáneas, y compris en Espagne, puisque durant les premières années du franquisme, étant donné la désorganisation du pays, le manque de papier, etc., ce sont les éditions sud-américaines, argentines en particulier, qui ont approvisionné le marché espagnol. Ainsi de El Amigo Manso il existe quatre éditions sudaméricaines entre 1939 et 1954, en plus de celle faite par Aguilar en 1951. 
œuvre théâtrale; dans le cas de Galdós aussi, il existe d'autres sources pour l'apprécier ${ }^{58}$.

Il ne faut cependant pas oublier que les succès des représentations a des retombées éditoriales, surtout à une époque où le théâtre était un phénomène beaucoup plus multitudinaire qu'aujourd'hui, le public de province cherchant à prendre connaissance de l'œuvre avant même qu'elle puisse y être représentée.

\section{On en a l'illustration avec le succès d'Electra.}

3.2.3.1. Le succès d'Electra. Cette pièce représentée en 1901, dans une atmosphère de scandale et d'anticléricalisme, passionna l'opinion -on le sait- pendant un bon moment, et les conséquences sur la vente de sa version imprimée sont évidentes ${ }^{59}$.

C'est ainsi qu'au palmarès des œuvres de Galdós, Electra figure en troisième position du point de vue des rythmes de vente. Cela s'explique, non par la durabilité de cette œuvre, mais par le fait qu'en 57 jours, de février à mars 1901 , ou en a vendu 27.000 exemplaires (c'est-à-dire presque autant que de Marianela de 1878 à la même date! $)^{60}$, soit un rythme de 473 exemplaires par jour; beaucoup plus que Pequeñeces, le dernier succès d'édition retentissant, 10 ans auparavant.

58 Par exemple, les chiffres de recettes de différentes représentations, des coupures de presse et de multiples lettres de spectateurs qui permettent de mettre en oeuvre une méthode inspirée de celle pratiquée par Noël Salomon, René Andioc, Jean Sentaurens, Jean Mouyen, François Sureda, etc.

59 Autant il est difficile de cerner le phénomène de la lecture d'oeuvres dramatiques (avant ou après avoir vu la représentation, après lecture d'une critique, etc.) - -il ne faut cependant pas oublier la clientèle que représentent les professionnels du théâtre-, dans le cas d'Electra, on voit très bien que les gens se sont précipités sur l'œuvre imprimée à la suite des échos nombreux qu'ils avaient pu lire dans la presse, l'occasion de voir la pièce représentée ne venant que plus tard, pour une partie d'entre eux seulement. Cette pratique reste néanmoins limitée, en raison des réticences du public à l'égard de la lecture de formes dramatiques, y compris des romans dialogués, comme le confirme, d'une certaine façon, cette observation de M. H. de Cámara à propos de La Loca de la casa: «un librero me dijo hace días que no había vendido ningún ejemplar del libro pues alguno que lo veía lo dejaba porque era comedia» (Lettre du 1602-1893 à Galdós (don Prisco). Archives de la Casa-Museo Pérez Galdós).

60 Pour donner un ordre d'idées, on dit que Nana a été vendu à 55.000 exemplaires le jour de sa parution. Sur les tirages des romans de Zola, voir Becker (Colette), Trente années d'amitié. Lettres de l'éditeur Georges Charpentier d̀ Emile Zola. 1872-1902, Paris, P. U. F., 1980. 
Ensuite, entre avril 1901 et 1903, le rythme tombe à 208 par mois (moins déjà que Mariucha à la même date), puis à 10 entre 1903 et 1920 et est de 13 entre 1920 et mars 1933 où un nouveau tirage de 4.000 exemplaires est effectué ${ }^{61}$.

Avec la configuration du succès -éphémère- d'Electra, on voit donc la nécessité de ne pas se contenter, pour une bibliométrie, du succès de chiffres globaux qui peuvent conduire à des erreurs d'appréciation graves.

3.2.3.2. Les autres œuvres dramatiques. Pour les autres œuvres, il ne se produit rien de comparable: Realidad atteint, 26 ans après sa première publication, le chiffre de 6.000 exemplaires imprimés (mais non forcément vendus). La Loca de la Casa au bout de 22 ans en est à 9.000 exemplaires et Mariucha, représentée et publiée en 1903, à 7.000 en 1921. De La de San Quintín, on a fait quatre éditions entre 1894 et 1906 et de La Fiera, trois de 1897 à 1920 , mais on sait que pour les œuvres dramatiques les éditions étaient en général de 2.000 exemplaires (3.000 au maximum) et les rythmes de vente ne sont pas conséquent pas très élevés.

D'après les données partielles disponibles, on voit ainsi que Realidad a, entre 1897 et 1904, un rythme de 7 et entre 1904 et 1906 de 2. Les rythmes de La loca de la casa, La de San Quintín, Voluntad et Doña Perfecta sont semblables ou inférieurs entre 1897 et 1904 à ce qu'on peut déduire de la date de leur réédition ${ }^{62}$.

Entre 1904 et 1906, le rythme de vente que connaît La Fiera (3) est bien au dessous de celui des romans en général, ce qui nous donne une mesure du succès éditorial des œuvres dramatiques, de façon courante.

61 Ces calculs sont faits à partir des indications sur les tirages successifs publiés dans la Relación de impresiones de la 3e édition. Il faut signaler également les éditions faites en 1901 en Argentine et au Mexique et les traductions en portugais et en hollandais cette même année. Electra semble être la seule pièce de Galdós à avoir été traduite du vivant de Galdós.

62 Les 432 exemplaires de La loca de la casa existant en 1897 sont épuisés en 1904, mais on n'en fait une nouvelle édition qu'en 1905, signe que la demande n'est pas très forte. On constate la même chose à propos de La de San Quintín. Voluntad et Doña Perfecta également épuisés en janvier 1904, mais dont les nouvelles éditions ne sont faites respectivement qu'en 1906. le 7-07-1907 (2.027 exemplaires) et en 1906. 
Si l'on observe le comportement des œuvres postérieures, dont la première représentation est encore récente en mai 1904, on voit à propos de Mariucha, par exemple, que si, de juillet 1903 au 15 janvier 1904, 2.981 exemplaires sont vendus (rythme de 426), de la nouvelle édition de 1.000 exemplaires faite en 1905 il reste encore, en mai 1906, 816 exemplaires, ce qui indique clairement une rupture dans le rythme de vente et permet d'estimer les lecteurs inconditionnels de l'œuvre dramatique de Galdós à une dizaine de milliers, toujours si l'on applique le coefficient de 3,5 au nombre d'exemplaires achetés ${ }^{63}$.

Quant à Bárbara et Amor y ciencia qui sont toutes les deux de 1905 , leur succès immédiat semble avoir été bien moindre, en particulier pour Bárbara, puisque, si on estime que le tirage de la première édition a été de 3.000 exemplaires, le rythme est de 46 pour Bárbara, pendant les treize premiers mois et de 148 pour Amor y ciencia durant les six premięrs. A partir de Bárbara, on observe d'ailleurs que les nouvelles ouvres dramatiques de Galdós éditées par Hernando ne connaissent pas de rééditions: pourtant le niveau de tirage reste identique et baisse même dans certains cas: 1.000 exemplaires pour Casandra en 1910 (avec une édition ultérieure de 2.000, mais en 1926), 3.000 pour Celia en los infiernos (1913) et Alceste (1914), 4.000 exceptionnellement pour El tacaño Salomón (1916), mais 1.000 seulement pour Santa Juana de Castilla (1918).

Il est dommage que les données concernant $E l$ abuelo, qui semble avoir connu un certain succès sur les planches, ne permettent pas d'apprécier un comportement éditorial marqué par des rééditions en 1913 et $1917 \mathrm{du}$... roman, car le succès du drame semble avoir bien davantage profité à la version romanesque qu'à la version dramatique.

3.2.3.3. Le comportement éditorial des ouvres dramatiques. Pour cette partie marginale de l'œuvre imprimée de Galdós, on peut dire que:

63 Les données concernant Alma y vida (2.916 exemplaires en magasin au 15-01-1904) ou sont fausses, ou se rapportent à une édition postérieure á celle de 1902 , la seule recensée par $\mathbf{M}$. Hernández Suárez, ou supposent un tirage beaucoup plus important que d'habitude. 
1. Mis à part le cas exceptionnel d'Electra, les œuvres dramatiques imprimées ne représentent pas un grand volume d'exemplaires et n'ont pas connu de succès d'édition (même à court terme) ${ }^{64}$.

2. Leur rythme de vente, pour autant qu'on puisse l'apprécier, semble être assez élevé mais chute de façon vertigineuse jusqu'à l'obsolescence de l'œuvre imprimée, quand bien même les représentations continuent.

3. Le succès d'édition semble avoir été de plus en plus faible de 1892 à 1918 et il serait intéressant de vérifier cette tendance par la mesure de la fréquentation.

3.3. La bourse des valeurs galdosiennes. Dans l'œuvre de Galdós on peut, de son vivant, distinguer deux grands groupes auxquels correspondent deux comportements éditoriaux bien différenciés ( $c f$. document 20 ):

1. Le premier groupe comprend la production romanesque des années 1873 à 1880 (1ère et 2ème séries des Episodios, Doña Perfecta, Gloria, Marianela et La Familia de León Roch; les autres œuvres sont difficilement saisissables) dont le succès initial, comparable à celui d'autres œuvres de l'époque, s'amenuise lentement jusqu'en 1904-1914 avec une différence entre le niveau de départ et le niveau d'arrivée de 3 à 1 .

2. Le second englobe la production postérieure à 1880: les Novelas Españolas Contemporáneas, avec des niveaux de départ semblables à ceux des romans du premier groupe, tombent très rapidement à des niveaux bien inférieurs, jusqu'à la léthargie; les trois dernières séries d'Episodios nacionales, à partir de niveaux de départ plus élevés que ceux du premier groupe, tombent très

64 C'est ce que dit très crûment à Galdós (don Prisco dans la correspondance) son éditeur M. H. de Cámara, dans une lettre du 6 mai 1893 où il fait un bilan très pessimiste de la marche de sa maison d'édition: «Realidad (drama) no ha cubierto gastos. La loca... (comedia), de ninguna de las dos se han cubierto gastos y algunos libreros han devuelto los ejemplares remitidos por no ser novelas y no tener compradores» (Archives de la Casa-Museo Pérez Galdós). 
rapidement au dessous de la moyenne du premier groupe; quant à la production dramatique qui est un cas un peu à part, à partir de niveaux bas, elle tombe rapidement dans l'obsolescence.

Après la mort de Galdós, le premier groupe bénéficie d'un certain regain de succès, la première série des $E$. N. en particulier, tandis que le seconde groupe, sauf cas exceptionnels et quelques variations, positives sous la Deuxième République, négatives sous le Franquisme, se maintient à son faible niveau de succès. Les années 1970 annoncent un regain général qui bénéficie proportionnellement plus au second groupe.

Il n'en reste pas moins que le succès le plus grand et le plus durable de Galdós lui vient de sa production de cinq à sept années seulement.

De cette manifestation commerciale du succès objectif de Galdós, se dégagent deux grandes lignes de force: une évidente hiérarchisation précoce des valeurs au sein même de son œuvre et une fluctuation de ces mêmes valeurs dans la longue durée.

Il faut maintenant essayer d'expliquer et d'interpréter ces deux tendances. 